Y. Yoshino

Nagoya Math. J.

Vol. 95 (1984), 41-50

\title{
ON NORTHCOTT-REES THEOREM ON PRINCIPAL SYSTEMS
}

\author{
YUJI YOSHINO
}

\section{§1. Introduction}

Let $R$ be a local ring with maximal ideal $m$ and let us make the following definition according to the paper [NR] of Northcott and Rees, which is essentially due to F. S. Macaulay.

Definition. A proper ideal $a$ of $R$ is said to be a principal system if, for any integer $N$, there exists an irreducible $\mathfrak{m}$-primary ideal $\mathfrak{q}$ satisfying $\mathfrak{a} \subset \mathfrak{q} \subset \mathfrak{m}^{N}+\mathfrak{a}$.

It will be worth noting that this definition is equivalent to saying that the ring $R / \mathfrak{a}$ is approximately Gorenstein, in the terminology of M. Hochster in $[\mathrm{H}]$.

In their paper [NR] Northcott and Rees obtained some fundamental properties of principal systems and proved the following

Theorem [NR; Theorem 6]. Let $R$ be a homomorphic image of a Gorenstein local ring. Then $\epsilon$ very ideal of $R$ is the intersection of a finite number of principal systems. (In [NR] it is assumed that $R$ is a homomorphic image of a regular local ring. However one can easily see that the same proof as in [NR] works successfully also in the case of a homomorphic image of a Gorenstein ring.)

The aim of this paper is to determine a perfect condition for rings to satisfy the conclusion of Northcott-Rees theorem. Our main result is;

Theorem. The following conditions are equivalent for a local ring $R$.

(1) Every ideal of $R$ is an intersection of a finite number of principal systems.

(2) Every irreducible ideal of $R$ is a principal system.

(3) Every prime ideal of $R$ is a principal system.

(4) If $\mathfrak{p} \in \operatorname{Spec}(R)$ and $\mathfrak{Q} \in \operatorname{Ass}_{\hat{R}}(\hat{R} / \mathfrak{p} \hat{R})$ such that $\operatorname{dim}(\hat{R} / \mathfrak{Q})=1$, then

Received November 12, 1982.

Revised September 29, 1983. 


$$
\operatorname{dim}_{k(\mathfrak{Q})} \operatorname{Hom}_{\hat{R}_{0}}\left(k(\Omega),(\hat{R} / \mathfrak{p} \hat{R})_{\mathfrak{\Omega}}\right)=1
$$

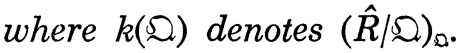

Indeed the equivalence of (1) and (2), and the implication from (2) to (3) are trivial. Other implications in Theorem will be discussed in section 2. $((3) \Rightarrow(4)$ and $(3) \Rightarrow(2)$ will be obtained from Proposition 4 and 5 respectively.)

By our theorem one can see that Northcott-Rees theorem holds in acceptable rings. (For the definition of acceptable rings, see [S].) In fact they satisfy the condition (4) in Theorem. But there exists an example of one-dimensional local domain in which Northcott-Rees theorem does not hold. We also have an example of two dimensional local domain which is not acceptable but in which Northcott-Rees theorem holds. Those examples are constructed in [FR] and we have only to check the condition (4) of Theorem for them. See section 3 for more discussion.

Throughout this paper all rings will be commutative Noetherian rings with identity.

\section{§2. Basic results on principal systems}

The name of "principal system" is probably derived from the following

Proposition 1. For a local ring $(R, \mathfrak{m}, k)$, the following are equivalent. (1) $R$ is an approximately Gorenstein ring.

(2) There exists a sequence $\left\{x_{n}\right\}_{n=1}^{\infty}$ of elements in $E_{R}(k)$, such that $R x_{1} \subset$ $R x_{2} \subset \cdots \subset R x_{n} \subset R x_{n+1} \subset \cdots \subset E_{R}(k)$ and $\bigcup_{n=1}^{\infty} R x_{n}=E_{R}(k)$, where $E_{R}(k)$ denotes the injective envelope of the residue field $k$ of $R$.

(3) For any $x, y \in E_{R}(k)$, there exists an element $z$ of $E_{R}(k)$ such that $R z \supset R x+R y$.

Proof. (1) $\Rightarrow(2)$ : If $R$ is approximately Gorenstein, then by the definition there is a set $\left\{\mathfrak{q}_{n}\right\}_{n=1}^{\infty}$ of irreducible m-primary ideals satisfying $\mathfrak{q}_{n} \subset \mathfrak{m}^{n}$ and $\mathfrak{q}_{n+1} \subset \mathfrak{q}_{n}$ for every $n$. Since $R / \mathfrak{q}_{n}$ is a Gorenstein ring of dimension 0 , the $R$-module $E_{\left(R / q_{n}\right)}(k) \cong\left[0: \mathfrak{q}_{n}\right]_{E_{R}(k)}$ is generated by one element, say $\left[0: \mathfrak{q}_{n}\right]_{E_{R}(k)}=R x_{n}(n=1,2,3, \cdots)$. Then it follows that $R x_{n} \subset$ $R x_{n+1}$. Furthermore we have $R x_{n} \supset\left[0: \mathrm{m}^{n}\right]_{E_{R}(k)}$ for every $n$. Hence $E_{R}(k)$ $=\bigcup_{n=1}^{\infty}\left[0: \mathfrak{m}^{n}\right]_{E_{R}(k)}=\bigcup_{n=1}^{\infty} R x_{n}$.

$(2) \Rightarrow(1)$ : If we denote $\mathfrak{q}_{n}=\left[0: x_{n}\right]_{R}$, then we have $R / \mathfrak{q}_{n} \cong R x_{n} \subset E_{R}(k)$. Thus every ideal $\mathfrak{q}_{n}$ is m-primary irreducible, for the submodule (0) of $E_{R}(k)$ is irreducible. It remains to prove that for each $n$ there exists an 
integer $N$ such that $\mathfrak{q}_{N} \subset \mathfrak{m}^{n}$. Since $\left[0: \mathfrak{m}^{n}\right]_{E_{R}(k)}$ is an $R$-module of finite length and $E_{R}(k)=\bigcup_{i=1}^{\infty} R x_{i}$, we have $\left[0: \mathfrak{m}_{n}\right]_{E_{R}(k)} \subset R x_{N}$ for sufficiently large $N$. Then $\mathfrak{q}_{N} \subset\left[0:\left[0: \mathfrak{m}^{n}\right]_{E_{R}(k)}\right]_{R}=\mathfrak{m}^{n}$.

The equivalence of (2) and (3) will be immediate if one notices that $E_{R}(k)$ is countably generated over $R$. (See [M: Theorem 3.11].)

A finitely generated module $K$ over a local ring $(R, \mathfrak{m}, k)$ is said to be a canonical module of $R$ if there is an isomorphism;

$$
K \otimes_{R} \hat{R}=\operatorname{Hom}_{\hat{R}}\left(H_{\mathrm{m}}^{d}(R), E(k)\right)
$$

where $d=\operatorname{dim}(R)$. Such a module $K$, if it exists, is uniquely determined by $R$ up to isomorphism. We refer the reader to [A] and [HK] for further information and details.

Proposition 2. If $R$ is a local ring which possesses the canonical module $K$, then $[0: x]_{R}$ is a principal system for any $x \in K$.

Proof. We may assume that $R$ is complete. If we denote $I=[0: x]_{R}$ for fixed $x \in K$, we have an injective map $f: R / I \rightarrow K$ by $f(1)=x$. Applying $\operatorname{Hom}_{R}\left(, E_{R}(k)\right)$ to $f$, we get a surjective homomorphism $g: H_{\mathrm{m}}^{d}(R) \rightarrow$ $\operatorname{Hom}_{R}\left(R / I, E_{R}(k)\right)$ where one should notice that $\operatorname{Hom}_{R}\left(R / I, E_{R}(k)\right) \simeq E_{(R / I)}(k)$.

On the other hand it is known that local cohomology modules are obtained by taking cohomology of Ceck complex, that is, if $\left\{a_{1}, a_{2}, \cdots, a_{d}\right\}$ is a system of parameters of $R$, then $H_{\mathrm{m}}^{i}(R)$ is an $i$-th cohomology module of the following complex;

$$
0 \longrightarrow R \longrightarrow \prod_{i=1}^{d} R_{a_{i}} \longrightarrow \cdots \longrightarrow \prod_{j=1}^{d} R_{a_{1} \cdots \hat{a}_{j} \cdots a_{d}} \longrightarrow R_{\left(a_{1} a_{2} \cdots a_{d}\right)} \longrightarrow 0 .
$$

In particular there is a surjective homomorphism $h: R_{y} \rightarrow H_{\mathrm{m}}^{d}(R)$ where $y=a_{1} a_{2} \cdots a_{d}$. If we consider the composition map $g \cdot h$, we also have a surjection of $R_{y}$ to $E_{(R / I)}(k)$. Let us denote $x_{n}=g \cdot h\left(1 / y^{n}\right) \in E_{(R / I)}(k)$ for $n=1,2,3, \cdots$. Since $R(1 / y) \subset R\left(1 / y^{2}\right) \subset \cdots \subset R\left(1 / y^{n}\right) \subset \cdots \subset R_{y}$ and $\bigcup_{n=1}^{\infty} R\left(1 / y^{n}\right)=R_{y}$, we also have $(R / I) x_{1} \subset(R / I) x_{2} \subset \cdots \subset(R / I) x_{n} \subset \cdots \subset$ $E_{(R / I)}(k)$ and $\bigcup_{n=1}^{\infty}(R / I) x_{n}=E_{(R / I)}(k)$. Hence by Proposition 1 we see that $I$ is a principal system.

Corollary. Let $R$ be a local ring possessing the canonical module $K$. If $R$ is unmixed and generically Gorenstein, then $R$ is approximately Gorenstein. 
Proof. Since $R$ is unmixed, we see that $\operatorname{Supp}_{R}(K)=\operatorname{Spec}(R)$. (See [A; (1.7)]. Thus [A; Corollary 4.3] shows that $K_{\mathfrak{p}}$ is a canonical module of $R_{\mathfrak{p}}$ for any prime ideal $\mathfrak{p}$. Then by the assumption one sees that $S^{-1} K$ $\simeq S^{-1} R$ where $S$ is the set of all non-zero divisors of $R$. Thus one can find an element $x$ of $K$ satisfying $[0: x]_{R}=(0)$. Hence $R$ is approximately Gorenstein by Proposition 2.

Next we would like to clarify the approximate Gorensteinness in one dimensional case. The following lemma will be useful for this purpose.

Lemma 1. Let $(R, \mathrm{~m}, k)$ be a local integral domain of dimension 1 which possesses a dualizing complex, and let $T$ be a finitely generated torsion-free $R$-module. If there exist an irreducible submodule $J$ of $T$ and an element $x(\neq 0)$ of $R$ such that

$$
x T \supset J \supset x^{n} T
$$

for some $n$, then $\operatorname{rank}(T)=1$.

Proof. Since $R$ has a dualizing complex, it also has a fundamental one (cf. $\left.\left[S_{2}\right]\right)$, thus there is an exact sequence;

$$
0 \longrightarrow K \longrightarrow Q(R) \longrightarrow E_{R}(k) \longrightarrow 0
$$

where $Q(R)$ is the field of fractions of $R$ and the finitely generated $R$ module $K$ is nothing but the canonical module of $R$. By the assumption $T / J$ is an $R$-module of finite length in which the zero submodule is irreducible. Hence it can be embedded into $E_{R}(k)$, and [we have the following diagram;

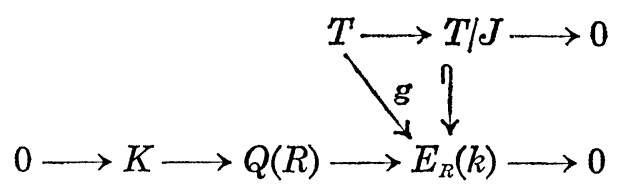

Applying $\operatorname{Hom}_{R}(T, \quad)$ to the sequence (*), we obtain the exact sequence;

$$
\operatorname{Hom}_{R}(T, Q(R)) \longrightarrow \operatorname{Hom}_{R}\left(T, E_{R}(k)\right) \longrightarrow \operatorname{Ext}_{R}^{1}(T, K),
$$

where $\operatorname{Ext}_{R}^{1}(T, K)$ should vanish by the local duality theorem, for $T$ is a Cohen-Macaulay module over a Cohen-Macaulay ring $R$. Therefore we can see that there exists $f \in \operatorname{Hom}_{R}(T, Q(R))$ which lifts $g$. Thus we have a commutative diagram; 


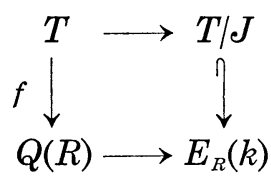

To prove the lemma, it suffices to see that $f$ is injective. For this purpose let us denote $\operatorname{Ker}(f)$ by $S$. Then by the above diagram $S \subset J \subset x T$. We claim that $S \subset x S$. In fact if $s \in S$, then $s$ can be written as a product $x \cdot t$ for some $t \in T$. Then $x f(t)=f(x t)=f(s)=0$, from which we have $f(t)=0$ since $x$ is a unit in $Q(R)$. Thus $t \in S$, hence $s=x \cdot t \in x S$. Therefore we get $S \subset x S$. Then Nakayama's lemma shows that $S=0$ as required.

Proposition 3. Let $R$ be a Cohen-Macaulay local ring of dimension 1 which has a dualizing complex. Then the following conditions are equivalent.

(1) $R$ is a generically Gorenstein ring.

(2) $R$ is approximately Gorenstein.

(3) There exist an m-primary irreducible ideal $I$ and an element $x$ of $\mathrm{m}$ satisfying $I \subset x R$.

Proof. $\quad(1) \Rightarrow(2)$ is already shown in Corollary to Proposition 2. It is also proved by $\mathrm{M}$. Hochster in $[\mathrm{H} ;(4.8 \mathrm{~b})]$.

$(2) \Rightarrow(3)$ is trivial.

$(3) \Rightarrow(1)$; Let $\mathfrak{p}$ be an arbitrary element of $\operatorname{Ass}(R)$ and put $T=[0: \mathfrak{p}]_{R}$. Then $T$ is a torsion-free $\bar{R}$-module, where $\bar{R}=R / \mathfrak{p}$. (In fact if $T$ has torsion over $\bar{R}$, then there exists $z(\neq 0) \in T \subset R$ such that $\mathfrak{m}^{N} z=0$ for large $N$. It therefore contradicts the fact $\operatorname{depth}(R)=1$.) Moreover if we denote $J=I \cap T$, then $J$ is an irreducible $\bar{R}$-submodule of $T$ and $x^{n} T \subset J$ for large $n$ since there is an injection of $T / J$ into $R / I$. On the other hand we can see the equality $x R \cap T=x T$. In fact if $x \cdot r \in x R \cap T(r \in R)$ then $\mathfrak{p} x r=0$, hence $\mathfrak{p} r=0$ since $x$ is not a zero divisor on $R$. Thus $r \in T$, and we have $x \cdot r \in x T$. In particular one sees that $J \subset x T$. Applying Lemma 1 to the $\bar{R}$-module $T$ we know that $T$ has rank 1 over $\bar{R}$, equivalently $T_{\mathfrak{v}}=\operatorname{Hom}_{R}(R / \mathfrak{p}, R)_{\mathfrak{p}} \simeq k(\mathfrak{p})$. This implies the Gorensteinness of $R_{p}$.

(Q.E.D.)

Corollary 1. Let $R$ be a Cohen-Macaulay local ring of dimension 1 which may not have a dualizing complex. Then $R$ is approximately Gorenstein if and only if $\hat{R}$ is generically Gorenstein. 
For the proof of this corollary we have only to notice that $R$ is approximately Gorenstein if and only if $\hat{R}$ is, and apply Proposition 1 to $\hat{R}$.

Corollary 2. Let $(R, \mathfrak{m})$ be a local ring and assume that, for every prime ideal $\mathfrak{p}$ of coheight 1 , the natural ring homomorphism of $R / \mathfrak{p}$ to $(R / \mathfrak{p})^{\wedge}$ is a Gorenstein homomorphism. If $\left\{\mathfrak{p}_{1}, \mathfrak{p}_{2}, \cdots, \mathfrak{p}_{n}\right\}$ is a set of prime ideals of coheight 1 such that $\mathfrak{p}_{i} \neq \mathfrak{p}_{j}(i \neq j)$, and $\mathfrak{a}_{i}$ is a $\mathfrak{p}_{i}$-primary irreducible ideal $(i=1,2, \cdots, n)$, then the ideal $\mathfrak{a}_{1} \cap a_{2} \cap \cdots \cap a_{n}$ is a principal system.

Proof. We may assume $\mathfrak{a}_{1} \cap \mathfrak{a}_{2} \cap \cdots \cap \mathfrak{a}_{n}=(0)$, i.e. $R$ can be supposed to be a Cohen-Macaulay ring of dimension 1 . Then we see that $R$ is generically Gorenstein if and only if $\hat{R}$ is generically Gorenstein by Lemma 2 below. Thus the conclusion is obtained from Corollary 1. (Notice that a $\mathfrak{p}$-primary ideal $\mathfrak{a}$ is irreducible if and only if $\operatorname{dim}_{k(\mathfrak{p})} \operatorname{Hom}_{R}$ $(R / \mathfrak{p}, R / \mathfrak{a})_{\mathfrak{p}}=1$.)

LEMma 2. Let $(R, \mathrm{~m})$ be a local ring and assume the following.

(1) For every $\mathfrak{p} \in \operatorname{Ass}(R), \operatorname{dim}_{k(\mathfrak{p})} \operatorname{Hom}_{R}(R / \mathfrak{p}, R)_{\mathfrak{p}}=1$.

(2) For every $\mathfrak{p} \in \operatorname{Ass}(R)$ and $\mathfrak{\beta} \in \operatorname{Ass}(\hat{R} / \mathfrak{p} \hat{R})$ satisfying $\operatorname{dim}(\hat{R} / \mathfrak{P})=1$,

$$
\operatorname{dim}_{k(\mathfrak{R})} \operatorname{Hom}_{\hat{R}}(\hat{R} / \mathfrak{R}, \hat{R} / \mathfrak{p} \hat{R})_{\mathfrak{\beta}}=1 .
$$

Then for every $\mathfrak{Q} \in \operatorname{Ass}(\hat{R})$ satisfying $\operatorname{dim}(\hat{R} / \mathfrak{Q})=1$, we have an equality;

$$
\operatorname{dim}_{k(\Omega)} \operatorname{Hom}_{\hat{R}}(\hat{R} / \Omega, \hat{R})_{\mathscr{Q}}=1 .
$$

In particular if $\operatorname{dim}(R)=1$, then $\hat{R}$ is a generically Gorenstein ring.

Proof. Let $\mathfrak{Q}$ be a prime ideal associated to $\hat{R}$ and $\operatorname{dim}(\hat{R} / \mathfrak{Q})=1$. Then we see that $\mathfrak{Q} \in \operatorname{Ass}(\hat{R} / \mathfrak{p} \hat{R})$ for some $\mathfrak{p} \in \operatorname{Ass}(R)$ since $\operatorname{Ass}(\hat{R})=$ $\bigcup_{\mathfrak{p} \in \operatorname{Ass}(R)} \operatorname{Ass}(\hat{R} / \mathfrak{p} \hat{R})$. By $\mathfrak{\supset} \hat{p} \hat{R}$ we have an injection;

$$
\operatorname{Hom}_{\hat{R}}(\hat{R} / \Omega, \hat{R}) \longrightarrow \operatorname{Hom}_{\hat{R}}(\hat{R} / \mathfrak{p}, \hat{R}) \simeq \operatorname{Hom}_{R}(R / \mathfrak{p}, R) \otimes_{R} \hat{R} .
$$

If we localize these $\hat{R}$-modules by $\mathfrak{Q}$, we get

$$
\operatorname{Hom}_{\hat{R}}(\hat{R} / \cong, \hat{R})_{\mathfrak{Q}} \longrightarrow \operatorname{Hom}_{R}(R / \mathfrak{p}, R)_{\mathfrak{p}} \otimes_{R \mathfrak{p}} \hat{R}_{\mathfrak{Q}} \simeq k(\mathfrak{p}) \otimes_{R \mathfrak{p}} \hat{R}_{\mathfrak{Q}} \simeq(\hat{R} / \mathfrak{p} \hat{R})_{\mathfrak{Q}}
$$

by the first assumption in the lemma. Thus the $\hat{R}$-module $\operatorname{Hom}_{\hat{R}}(\hat{R} / \Omega, \hat{R})_{\mathfrak{Q}}$ can be embedded into $\operatorname{Hom}_{\hat{R}}(\hat{R} / \mathfrak{Q}, \hat{R} / \mathfrak{p} \hat{R})_{\Re} \simeq k(\Omega)$, and hence it completes the proof.

Proposition 4. If a prime ideal $\mathfrak{p}$ of $R$ is a principal system and 


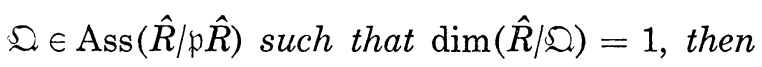

$$
\operatorname{dim}_{k(\Omega)} \operatorname{Hom}_{\hat{R}}(\hat{R} / \cong, \hat{R} / \mathfrak{h} \hat{R})_{\Omega}=1 .
$$

Proof. We may assume that $p=(0)$, i.e. $R$ may be supposed to be an approximately Gorenstein domain. Let us denote $\bar{R}=\hat{R} / \mathfrak{Q}$ and $T=$ [0: $]_{\hat{R}}$. Then $T$ is a torsion-free module over a one-dimensional local domain $\bar{R}$ as in the proof of Proposition 3. For every integer $N$, there exists an m-primary irreducible ideal $I_{N}$ of $\hat{R}$ such that $I_{N} \subset \mathfrak{m}^{N} \hat{R}$. If we denote $J_{N}=I_{N} \cap T$ and if we take arbitrary $x \in \mathfrak{m} \hat{R} \backslash \mathfrak{\Omega}$, then $J_{N}$ is irreducible in $T$ and $T / J_{N}$ is of finite length over $\bar{R}$ for every $N$. Moreover, if $N$ is sufficiently large then $\mathrm{m}^{N} \hat{R} \cap T \subset x T$ by Artin-Rees lemma, therefore $J_{N}$ is contained in $x T$. Thus Lemma 1 shows that the rank of $T$ over $\bar{R}$ is one, as required.

By virtue of Lemma 2, in order to prove the implication from (4) to (2) in Theorem, it suffices to verify the following

Proposition 5. Let $R$ be a local ring which has a dualizing complex and $\operatorname{depth}(R)>0$, and assume that for every $\mathfrak{p} \in \operatorname{Ass}(R)$ of $\operatorname{dim}(R / \mathfrak{p})=1$, $\operatorname{dim}_{k(\mathfrak{p})} \operatorname{Hom}_{R \mathfrak{p}}\left(k(\mathfrak{p}), R_{\mathfrak{p}}\right)=1$. Then $R$ is approximately Gorenstein.

Remark. This result is contained in a theorem of $\mathrm{M}$. Hochster in $[\mathrm{H}$; (1.6)]. But for the completeness of this paper we shall show a brief proof below, using our proposition 1.

Proof. We proceed by induction on $\operatorname{dim}(R)$. If $\operatorname{dim}(R)=1$, then $R$ is Cohen-Macaulay and generically Gorenstein by the hypothesis. The consequence is hence obtained from Corollary to Proposition 2.

Assume that $\operatorname{dim}(R) \geqq 2$. Notice that one can assume $R$ is a complete local ring. In fact, for any $\supseteq \in \operatorname{Ass}(\hat{R})$ such that $\operatorname{dim}(\hat{R} / \mathfrak{\Omega})=1$ there is a prime ideal $\mathfrak{p} \in \operatorname{Ass}(R)$ satisfying $\mathfrak{Q} \in \operatorname{Ass}(\hat{R} / \mathfrak{p} \hat{R})$. Since $R / \mathfrak{p}$ is an acceptable $\operatorname{ring}\left(\left[S_{2}\right]\right)$, if $\operatorname{dim}(R / \mathfrak{p}) \geqq 2$, then no prime ideal in $\operatorname{Ass}(\hat{R} / \mathfrak{p} \hat{R})$ has coheight 1. For the Gorensteinness of the formal fibers shows that every prime ideal in $\operatorname{Ass}(\hat{R} / \mathfrak{p})$ is a minimal prime of $\hat{R} / \mathfrak{p} \hat{R}$ and therefore if there were a prime ideal $\mathfrak{Q}^{\prime} \in \operatorname{Ass}(\hat{R} / \mathfrak{p} \hat{R})$ such that $\operatorname{dim}\left(\hat{R} / \mathfrak{Q}^{\prime}\right)=1$, then $R / \mathfrak{p}$ would not be quasi-unmixed unless $\operatorname{dim}(R / \mathfrak{p})=1$. However it never happen since $R$ is universally catenary. (See $[R]$.) Thus one obtains $\operatorname{dim}(R / \mathfrak{p})=1$ and hence $\operatorname{dim}_{k(\mathfrak{p})} \operatorname{Hom}_{R}(R / \mathfrak{p}, R)_{\mathfrak{p}}=1$ by the assumption.

On the other hand one can see that the formal fiber $(\hat{R} / \mathfrak{p} \hat{R})_{\Omega}$ is a Gorenstein ring of dimension 0 , since $R$ is acceptable. In other words, 
$\operatorname{dim}_{k(\mathfrak{\Omega})} \operatorname{Hom}_{\hat{R}}(\hat{R} / \Omega, \hat{R} / \mathfrak{p} \hat{R})_{\Omega}=1$. Then in the same way of the proof of Lemma 2, we see that $\operatorname{dim}_{k(\Omega)} \operatorname{Hom}_{\hat{R}}(\hat{R} / \Omega, \hat{R})_{\mathfrak{\Omega}}=1$. Thus $\hat{R}$ satisfies the same condition as $R$ and we may hence assume the completeness of $R$.

By Proposition 1 it is sufficient to see that for every finitely generated $R$-submodule $M$ of $E_{R}(k)$ there exists an element $x$ of $E_{R}(k)$ such that $M \subset R x$. For this purpose let $(0)=\mathfrak{q}_{1} \cap \mathfrak{q}_{2} \cap \cdots \cap \mathfrak{q}_{n}$ be an irredundant decomposition of $(0)$ in $R$ into irreducible ideals and let $\mathfrak{p}_{i}=\sqrt{\mathfrak{q}_{i}}(i=1$, $2, \cdots, n)$. By the assumption if $\operatorname{dim}\left(R / \mathfrak{p}_{i}\right)=\operatorname{dim}\left(R / \mathfrak{p}_{j}\right)=1$ for some $i \neq j$, then $\mathfrak{p}_{i} \neq \mathfrak{p}_{j}$. Thus we can find a set $\left\{\mathfrak{P}_{1}, \mathfrak{P}_{2}, \cdots, \mathfrak{P}_{n}\right\}$ of prime ideals satisfying the following conditions;

$$
\begin{aligned}
& \operatorname{dim}\left(R / \mathfrak{P}_{i}\right)=1 \quad(i=1,2, \cdots, n) . \\
& \mathfrak{P}_{i} \neq \mathfrak{P}_{j} \quad \text { if } i \neq j . \\
& \mathfrak{P}_{i} \supset \mathfrak{P}_{i} \quad(i=1,2, \cdots, n) .
\end{aligned}
$$

Since $\bigcap_{i=1}^{n} \bigcap_{j=1}^{\infty}\left\{\left(\mathfrak{P}_{i}^{j} R_{\mathfrak{F}_{i}}+\mathfrak{q}_{i} R_{\mathfrak{\Re}_{i}}\right) \cap R\right\}=\bigcap_{i=1}^{n}\left(\mathfrak{q}_{i} R_{\mathfrak{\Re}_{i}} \cap R\right)=(0)$ and since $M$ is an $R$-module of finite length, we have that $\bigcap_{i=1}^{n}\left\{\left(\mathfrak{P}_{i}^{N} R_{\mathfrak{P}_{i}}+\mathfrak{q}_{i} R_{\mathfrak{F}_{i}}\right) \cap R\right\}$. $M=(0)$ for large enough $N$ by the theorem (30.1) in [N]. Each ring $\bar{R}_{i}:=R_{\mathfrak{P}_{i}} / \mathfrak{q}_{i} R_{\mathfrak{P}_{i}}$ has a dualizing complex and the zero ideal of $\bar{R}_{i}$ is irreducible. Thus the induction hypothesis shows that $\bar{R}_{i}$ is approximately Gorenstein for $i=1,2, \cdots, n$. It follows that there exists a $\mathfrak{P}_{i} \bar{R}_{i}$-primary and irreducible ideal $\mathfrak{\Omega}_{i}^{\prime}$ of $\bar{R}_{i}$ such that $\mathfrak{Q}_{i}^{\prime} \subset \mathfrak{P}_{i}^{N} \bar{R}_{i}(i=1,2, \cdots, n)$. If we denote $\mathfrak{Q}_{i}=\mathfrak{Q}_{i}^{\prime} \cap R$ and $\mathfrak{Q}=\mathfrak{Q}_{1} \cap \mathfrak{Q}_{2} \cap \cdots \cap \mathfrak{Q}_{n}$, then each $\mathfrak{\bigcap}_{i}$ is $\mathfrak{P}_{i}$-primary and irreducible and $\mathfrak{\bigcap}_{i} \subset\left(\mathfrak{P}_{i}^{N} R_{\mathfrak{P}_{i}}+\mathfrak{q}_{i} R_{\mathfrak{P}_{i}}\right) \cap R(i=1,2, \cdots, n)$. Hence we see that $\mathfrak{Q} \cdot M=(0)$ and $R / \cong$ is a Cohen-Macaulay local ring of dimension 1 which is generically Gorenstein (and has a dualizing complex). In particular $R / \cong$ is approximately Gorenstein by Corollary to Proposition 2. Notice that there exists an isomorphism $E_{(R / \Omega)}(k) \simeq$ [0: $\cong]_{E_{R}(k)}$, and therefore $M$ can be considered as a submodule of $E_{(R / \Omega)}(k)$. Since $R / \bigcap$ is approximately Gorenstein, we can find an element $x \in$ [0: $\mathfrak{l}_{E_{R}(k)} \subset E_{R}(k)$ satisfying $R x \supset M$ by Proposition 1 . This completes the proof.

\section{$\S 3 . \quad$ Examples}

We shall give two examples below. Such bad Noetherian local rings are constructed by Ferrand and Raynaud in [FR]. Hence for the detail of construction we refer the reader to their paper.

Example 1. There exists a local integral domain of dimension 1, in 
ih Northcott-Rees theorem does not hold.

In fact for an arbitrary integer $r \geqq 0$, there is a one-dimensional 1 domain $R$, such that $\hat{R}$ possesses a unique minimal prime ideal $\mathfrak{\beta}$ ch satisfies $\mathfrak{P}^{2}=(0)$ and $\mathfrak{P} \simeq(R / \mathfrak{B})^{r}$. [FR; Proposition 3.1]. If $r \geqq 2$, I such a ring $R$ does not satisfy the condition (4) in Theorem, since ${ }_{k(\mathfrak{R})} \operatorname{Hom}_{\hat{R}}(\hat{R} / \Re, \hat{R})=r$.

ExAmple 2. There exists a local integral domain of dimension 2, which Northcott-Rees theorem holds, but whose completion has an sedded prime ideal. In particular it is neither acceptable nor excellent. Proposition 3.3 in [FR] and its proof show that there is a local domain if dimension 2 such that

$$
\hat{R}=C[[X, Y, Z]] /\left(Z^{2}, t Z\right)
$$

sre $t=X+Y+Y^{2} s$ for some $s \in C[[Y]] \backslash C\{Y\}$. Let us denote $\mathfrak{B}=$ $t) \hat{R}$ and $\mathfrak{Q}=Z \hat{R}$. Notice that they are prime and $\mathfrak{\complement} \mathfrak{R}$. Since $=(Z) \cap\left(Z^{2}, t\right)$ is a primary decomposition of $(0)$ in $\hat{R}$, we have $\operatorname{Ass}(\hat{R})$

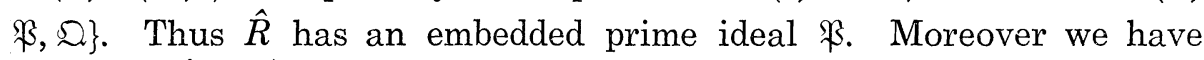
$\iota_{k(\mathfrak{R})} \operatorname{Hom}_{\hat{R}}(\hat{R} / \mathfrak{R}, \hat{R})_{\Re}=1$, for $\left(Z^{2}, t\right)$ is $\mathfrak{\beta}$-primary and irreducible.

In order to prove that $R$ satisfies the condition (4) in Theorem, it ices to see that, for every prime ideal $\mathfrak{p}$ of $R$ of height $1, \hat{R} / \mathfrak{p} \hat{R}$ is 1erically Gorenstein. If we take $\mathfrak{P}^{\prime} \in \operatorname{Ass}(\hat{R} / \mathfrak{p} \hat{R})$, then it can be seen $\not \supset\left(Z^{2}, t\right)$. In fact if $\mathfrak{P}^{\prime} \supset\left(Z^{2}, t\right)$, then $\mathfrak{P}^{\prime}=\mathfrak{B}$ and $(0)=\mathfrak{B} \cap R=\mathfrak{P}^{\prime} \cap R$ $p$, which is a contradiction. From this fact we obtain that $\hat{R}_{\mathfrak{F}^{\prime}}$ is a ular local ring, in particular it is Gorenstein. Therefore $(\hat{R} / \mathfrak{p} \hat{R})_{\mathfrak{P}^{\prime}}$ is o Gorenstein, since it is a fiber of a faithfully flat homomorphism of to $\hat{R}_{\mathfrak{B}^{\prime}}$. This is what we wanted.

\section{REFERENCES}

Y. Aoyama, Some basic results on canonical modules, J. Math. Kyoto Univ., 23 no. 1 (1983), 85-94.

i] D. Ferrand and M. Raynaud, Fibres formelles d'un anneau local noethérien, Ann. Sci. École Norm. Sup. (4), t. 3 (1970), 295-311.

M. Hochster, Cyclic purity versus purity in excellent Noetherian ring's, Trans. Amer. Math. Soc., 231 no. 2 (1977), 463-488.

1] J. Herzog, E. Kunz et al., Der kanonische Modul eines Cohen-Macaulay Rings, Lect. Notes in Math., 238, Springer Verlag, (1971).

E. Matlis, Injective modules over Noetherian rings, Pacific J. Math., 8 (1958), 511-528.

M. Nagata, Local Rings, Interscience Tracts in Pure and Applied Math., 13, J. Wiley, New York, 1962. 
[NR] D. G. Northcott and D. Rees, Principal systems, Quart. J. Math. Oxford (2), 8 (1957), 119-127.

[R] L. J. Ratliff, On quasi-unmixed local domains, the altitude formula, and the chain condition for prime ideals (I), Amer. J. Math., 91 (1969), 508-528.

[S] R. Y. Sharp, Acceptable rings and homomorphic images of Gorenstein rings, J. Algebra, 44 (1977), 246-261.

[S $\left.\mathrm{S}_{2}\right]$ R. Y. Sharp, A commutative Noetherian rings which possesses a dualizing complex is acceptable, Math. Proc. Cambridge Philos. Soc., 82 (1977), 197-213.

Department of Mathematics

Faculty of Science

Nagoya University

Chikusa-ku, Nagoya 464

Japan 